\title{
Asteroseismic signatures of helium gradients in main-sequence A stars; application to the roAp star HD 60435
}

\author{
S. Vauclair ${ }^{1}$ and $\mathrm{S}$. Théado ${ }^{1,2}$ \\ ${ }^{1}$ Université Paul Sabatier, Observatoire Midi-Pyrénées, CNRS/UMR5572, 14 av. E. Belin, 31400 Toulouse, France \\ e-mail: svcr@obs-mip.fr \\ 2 Centro de Astrofisica da Universidade do Porto, rua das Estrelas, 4150-762 Porto, Portugal
}

Received 8 January 2004 / Accepted 28 May 2004

\begin{abstract}
Asteroseismology is found to be a excellent tool for detecting diffusion-induced helium gradients inside mainsequence A stars. Models have been computed for 1.6 and 2.0 $M_{\odot}$ stars with pure helium diffusion, at different ages, so that the helium gradient lies at different depths inside the star. The adiabatic oscillation frequencies have been analysed and compared with those of a model without diffusion. Clear signatures of the diffusion-induced helium gradient are found in the so-called "second differences": these frequency differences present modulations due to the partial reflexion of the sound waves on the layer where the helium gradient occurs. A tentative application to the roAp star HD 60435, which presents enough detected oscillation frequencies for the test to be possible, is very encouraging. The results suggest the presence of a helium gradient inside the star, which is consistent with the idea that the triggering of the oscillations is due to the hydrogen $\kappa$-mechanism.
\end{abstract}

Key words. stars: oscillations - stars: abundances - diffusion - stars: chemically peculiar

\section{Introduction}

It is now widely recognized that the abundance anomalies observed in peculiar A stars are basically due to element diffusion. Those for which the radiative acceleration is larger than gravity (like most of the metals) are pushed upwards while the others, such as helium, diffuse downwards (Michaud 1970; Vauclair \& Vauclair 1982). The resulting atmospheric abundances depend on the competition between element diffusion and macroscopic motions like convection, mass loss, rotation-induced mixing and other kinds of mixing processes. Among the peculiar A stars, those which have large organized magnetic fields (the socalled Ap stars) are more complex than the others (like Hg-Mn stars): they show evidence of abundance spots or rings modulated by the magnetic structure. Among the coolest Ap stars (effective temperatures between $6700 \mathrm{~K}$ and $8700 \mathrm{~K}$ ) some oscillate with periods of a few minutes (the roAp stars) while the others seem stable (noAp stars). In roAp stars, the amplitudes of the oscillation modes are modulated according to the rotation period, which is explained in the framework of the oblique pulsator model (Kurtz 1990).

The helium $\kappa$-mechanism was invoked in the past as a possible way to trigger the oscillations: a well-adjusted competition between helium settling and mass loss could increase the helium abundance in stellar atmospheres as observed in helium-rich stars (Vauclair 1975, Vauclair et al. 1991). However, recent papers (Dziembowski \& Goode 1996; Balmforth et al. 2001) show that, even in this case, helium is not able to destabilize the star. On the other hand they find that, in some cases, the oscillations in A stars can be triggered by the hydrogen $\kappa$-mechanism. Such a process may be enhanced by the settling of helium, which diffuses downwards in the absence of strong mass loss.

In the present paper we study the asteroseismic signature of a helium gradient inside main-sequence A-type stars. As mentioned by Gough (1990), rapid variations of the sound velocity inside a star lead to partial reflections of the sound waves, which may clearly appear as frequency modulations in the socalled "second differences":

$\delta_{2} v_{n, l}=v_{(n+1), l}+v_{(n-1), l}-2 v_{n, l}$

which are computed for the same value of the azimutal number $l$. The modulation period of the oscillations is twice the "acoustic depth" of the region where the feature occurs (i.e. the time needed for the sound waves to travel between this region and the stellar surface).

Such an effect has been extensively studied in the literature for stellar models in which helium settling is neglected. In this case, the modulations in the frequencies are mostly due to the HeII ionisation zone and to the edge between the convective and radiative zones (Monteiro \& Thompson 1998; Roxburgh \& Vorontsov 2001; Mazumdar \& Antia 2001; Miglio et al. 2003)

We show here that the presence of a diffusion-induced helium gradient leads to a kink in the sound velocity with a very clear signature in the oscillation frequencies: asteroseismic observations of A-type stars can test helium diffusion and lead 
to a precise value of the acoustic depth corresponding to the position of the helium gradient inside the star.

For the present study, we neglect the effects of magnetic fields (the stars are assumed spherically symetric) and we do not compute the diffusion of heavy elements. Complete computation of element diffusion including the radiative acceleration on metals is a demanding work, especially for these stars where heavy element diffusion occurs in the atmosphere, so that radiative transfer should be solved in detail in the optically thin regions. This is out of the scope of the present paper, where we are interested in the structural changes induced by helium settling below the stellar surface and its influence on the oscillation frequencies. It has recently been shown (e.g. Richard et al. 2001) that in case of pure diffusion iron should accumulate deeper inside the stars, at temperatures around $200000 \mathrm{~K}$, where it can create a small convective zone. As the results we obtain here are very encouraging, new computations including heavy element diffusion should be done in the near future to see whether such a feature may be checked with asteroseismology.

We apply this test to the only roAp star in which enough modes have been observed, namely HD 60435 (Matthews et al. 1987). The results are extremely encouraging and show the importance of detecting as many modes as possible in rapidly oscillating Ap stars.

\section{Theoretical discussion}

\subsection{Seismic signatures of helium gradients}

Stellar acoustic $p$ modes with low $l$ values can propagate deeply inside the stars. For this reason, they may be used to obtain information on the deep stellar structure. However, in the case of strong gradients in the sound velocity, which may be due to the boundary of a convective zone, to the helium ionization region, or to helium gradients, the waves are partly reflected: this creates modulations in the frequency values which may be clearly visible in the second differences. The modulation periods are equal to $2 t_{s}$ where $t_{s}$ is the time needed for the acoustic waves to travel between the surface and the considered region (acoustic depth), i.e.:

$t_{s}=\int_{r_{s}}^{R} \frac{\mathrm{d} r}{c(r)}$

where $c(r)$ is the sound velocity at radius $r$, and $r_{s}$ the radius of the considered region.

As the $l$ dependence of the oscillatory signal is small for high frequency modes of low degrees (Monteiro et al. 1994), computational results for modes of different low $l$ values can be treated together. Here we show that the presence of diffusioninduced helium gradients lead to very clear signatures in the modulation of the second differences.

\subsection{Models with helium diffusion}

We have chosen to study the evolution of $1.6 M_{\odot}$ and $2.0 M_{\odot}$ stellar models, with pure helium settling. The stellar evolution code we use is the "Toulouse-Geneva code" which has been described many times in the literature (e.g. Richard et al. 1996;
Table 1. Model parameters.

\begin{tabular}{lrccrll}
\hline \hline $\begin{array}{l}\text { Mass } \\
\left(M_{\odot}\right)\end{array}$ & $\begin{array}{r}\text { Age } \\
(\mathrm{Myr})\end{array}$ & $\log \left(L / L_{\odot}\right)$ & $T_{\text {eff }}$ & $\begin{array}{r}t_{\star} \\
(\mathrm{s})\end{array}$ & $\begin{array}{l}t_{s} \\
(\mathrm{~s})\end{array}$ & $r_{s} / R$ \\
\hline 1.6 & 95 & 0.84 & 7936 & 5400 & 1480 & 0.93 \\
1.6 & 591 & 0.88 & 7585 & 6920 & 2700 & 0.86 \\
1.6 & 1600 & 1.05 & 7071 & 11240 & 5160 & 0.80 \\
$1.6 \mathrm{~h}$ & 1600 & 1.06 & 7352 & 10227 & & \\
2.0 & 63 & 1.22 & 9521 & 5917 & 1470 & 0.94 \\
2.0 & 649 & 1.31 & 8293 & 10150 & 4100 & 0.85 \\
\hline
\end{tabular}

Note. $t_{\star}$ represents the total acoustic radius of the models, $t_{s}$ the acoustic depth at the location of the helium gradients and $r_{s} / R$ the corresponding fractional radii; all the models presented in this table include helium diffusion, except the model labelled 1.6h which is homogeneous in its outer layers.

Théado \& Vauclair 2003). The physical input and parameters are those described in Richard, Théado \& Vauclair (2004): they include the most recent studies available for the equation of state, opacities and nuclear reaction rates. Helium diffusion is treated as described in these papers (see also Vauclair 2003). Metal diffusion is not included here and no mixing process or mass loss is taken into account. In real stars, there must be some mixing which prevents element diffusion from producing extreme abundance anomalies. Computations including mixing as well as metal diffusion will be done in the near future. The aim of the present paper is to study the precise signature on the oscillation frequencies of helium gradients inside stars.

We have computed the oscillation frequencies and their second differences in five models: $1.6 M_{\odot}$ with ages $95 \mathrm{Myr}$ and $591 \mathrm{Myr}$, and 1.6 Gyr and 2.0 $M_{\odot}$ with ages $63 \mathrm{Myr}$ and 649 Myr (Table 1). The results are given in Figs. 1 to 5. Only modes of degrees $l=0,1,2$ and 3 have been taken into account so that they could be treated together for the study of the second differences. The oscillation frequencies have been limited to the range 0.5 to $2 \mathrm{mHz}$ to take into account the asymptotic approximation validity and the stellar cut-off. In this range 80 to $100 \mathrm{~s}$ differences are computed, according to the model.

In each figure six graphs are displayed: a) the helium profile inside the star; b) the sound velocity, which shows a clear kink at the place of the helium gradient; c) the first derivative of the sound velocity in which the kink is still clearer; d) the second differences which show periodical oscillations; e) the Fourier transform of these oscillations, in which clear peaks are found for precise time values; f) the time needed for the acoustic waves to travel between the surface and the considered radius, or "acoustic depth".

We obtain clear signatures in the second differences of the frequencies of the diffusion-induced helium gradients inside stars. It can be checked that the periods corresponding to the peaks in the Fourier transforms (graphs e) are exactly twice the acoustic depths of the helium gradients (graphs f), as expected. In these figures, the scales are all the same except for graphs d) and f). In particular the Fourier transforms (graphs e) are presented at the same scale in each figure to show how the peak 

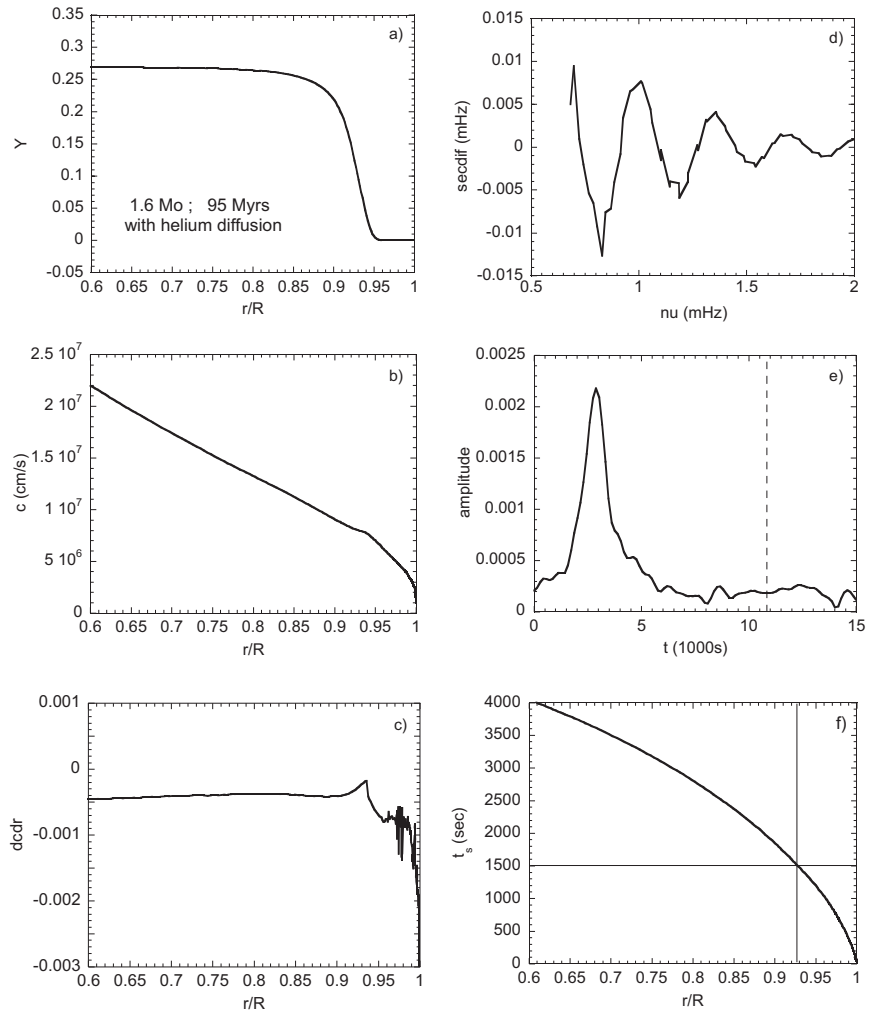

Fig. 1. Helium diffusion and its consequences for the stellar structure and oscillation frequencies for a $1.6 M_{\odot}$ at $95 \mathrm{Myr}$; a) helium profile as a function of the fractional radius; b) sound velocity: a kink is clearly visible at the place of the helium gradient; c) first derivative of the sound velocity: the kink is still more visible; $\mathbf{d}$ ) the second differences of the oscillation frequencies plotted as a function of the frequencies; e) the Fourier transform of graph d) plotted as a function of time (in thousands of seconds); the vertical dashed line corresponds to twice the total acoustic depth of the star (see Table 1); f) the "acoustic depth", or time needed for the acoustic waves to travel from the surface to the considered radius: it can be checked that the peak of graph e) corresponds to a time scale which is twice the "acoustic depth" of the helium gradient.

amplitudes decrease for deeper layers (larger times). As shown by Mazumdar \& Antia (2001), the amplitude of the oscillatory signal in the second differences contains an amplification factor of $4 \sin ^{2}\left(\pi t_{s} / t_{*}\right)$ where $t_{*}$ is the total acoustic radius of the star and $t_{s}$ the acoustic depth of the partial reflection region. Here this factor increases from about 2 to about 4 as the models evolve. The amplitude decrease by a factor $\simeq 10$ obtained in the present computations must be due to a competing process. It may be due partly to the fact that the energy in $p$ waves decreases towards the center and partly to the fact that the helium gradient becomes smoother with age, so that the reflected part (oscillations) is smaller for deeper discontinuities.

With these figures we can clearly follow the signatures of the helium gradients as they sink into the stars. The corresponding ages are obtained with the assumption of pure diffusion. In real stars macroscopic effects may change the time scales for helium settling. Comparisons with observed frequencies will give the positions of helium gradients inside the star, but we
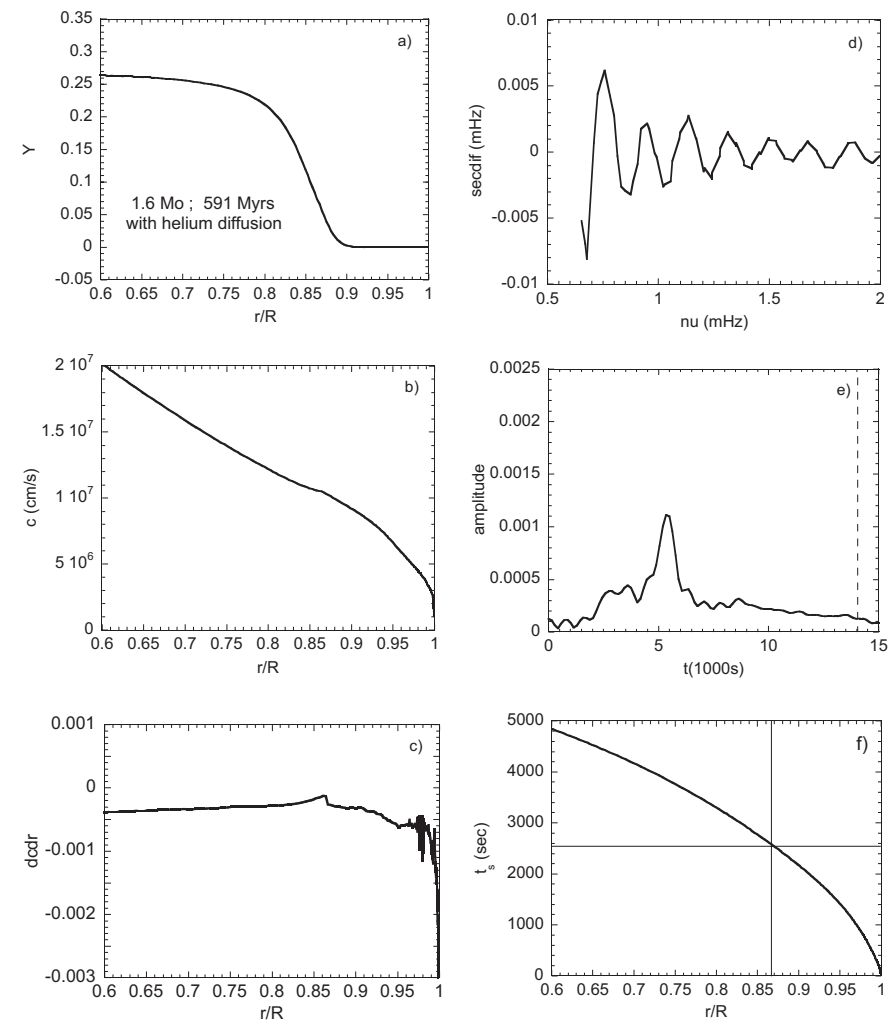

Fig. 2. Same as Fig. 1, for a $1.6 M_{\odot}$ model at $591 \mathrm{Myr}$.

must keep in mind that the time needed for these gradients to develop depends on the physics.

\subsection{Models without element diffusion}

Similar computations have been done for evolution models in which helium diffusion is suppressed. In this case no helium gradient exists in the stellar outer layers and the oscillatory signal described in the previous section is not present. However, helium ionization induces a typical variation of the $\Gamma_{1}$ coefficient which gives rise to another type of oscillatory signal in the frequencies. Computational results are shown in Fig. 6 for the case of the $1.6 M_{\odot}$ model at $1.6 \mathrm{Gyr}$. In this case graph a) displays the $\Gamma_{1}$ coefficient in the stellar outer layers. As already studied by several authors (e.g. Miglio et al. 2003), this coefficient is strongly influenced by the helium ionisation gradients. We can see the corresponding kinks in the sound velocity and its first derivative. This oscillatory signal does not appear when diffusion is effective as helium is then depleted in the region considered. Note that in the graphs of Fig. 6 the scales are quite different from those of Figs. 1 to 5. The effect due to the helium ionization gradients is much smaller than the effect due to the overall helium gradient in the case of diffusion. Here the amplification factor $4 \sin ^{2}\left(\pi t_{s} / t_{*}\right)$ is about 10 times smaller for the homogeneous model than for the model of the same age which includes helium diffusion. The large difference between the signal amplitudes in the two cases may be due to this effect. The right-hand graphs display the second differences, their Fourier transform and the travel time of the waves, as in 

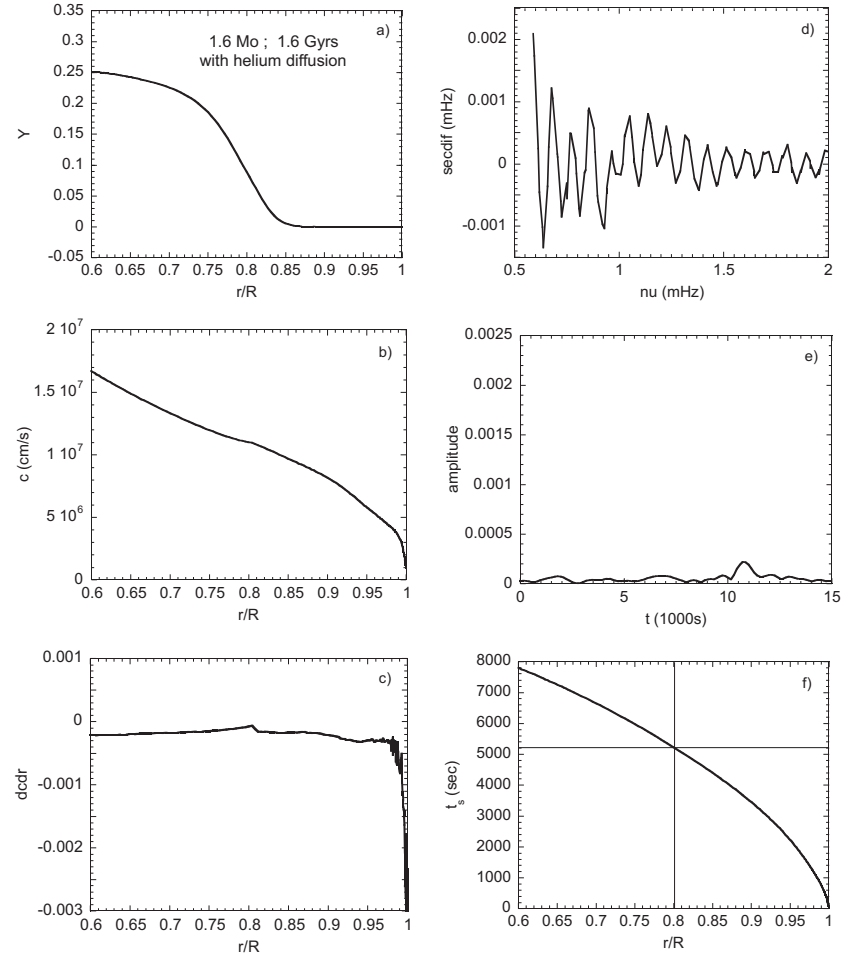

Fig. 3. Same as Fig. 1, for a $1.6 M_{\odot}$ model at $1.6 \mathrm{Gyr}$.
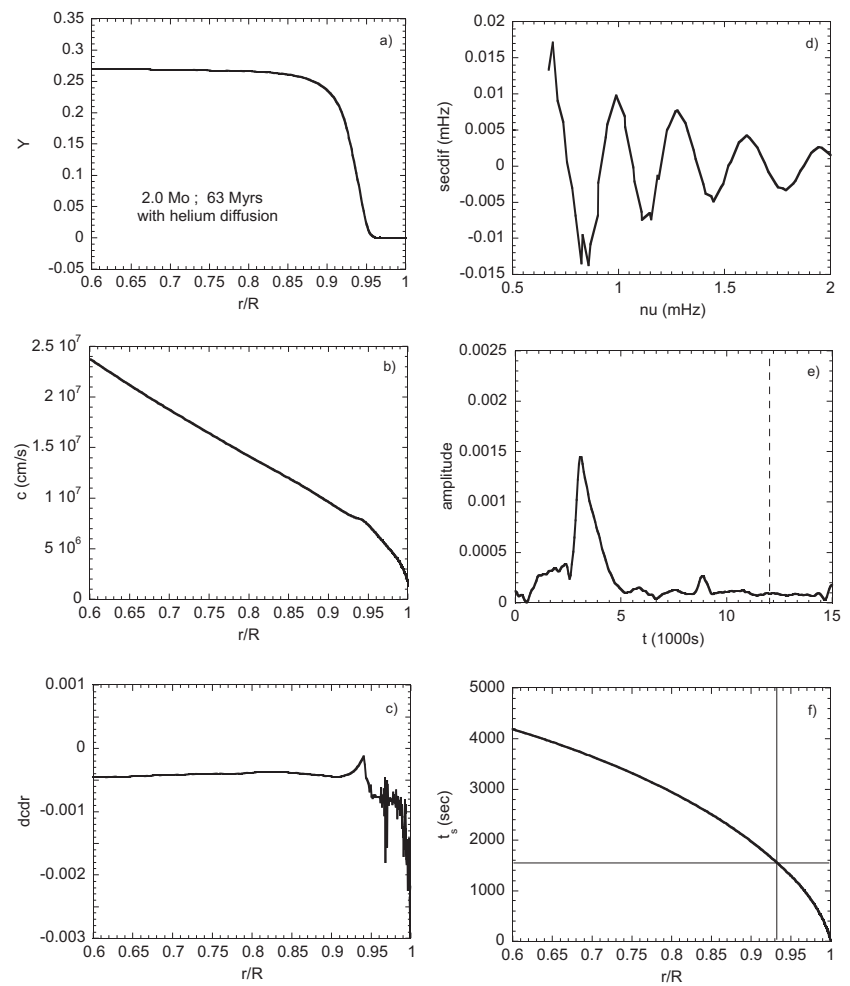

Fig. 4. Same as Fig. 1, for a $2.0 M_{\odot}$ model at $63 \mathrm{Myr}$.

Figs. 1 to 5 . The features are very different from those obtained in case of diffusion, where the signature of the helium gradient is dominant.
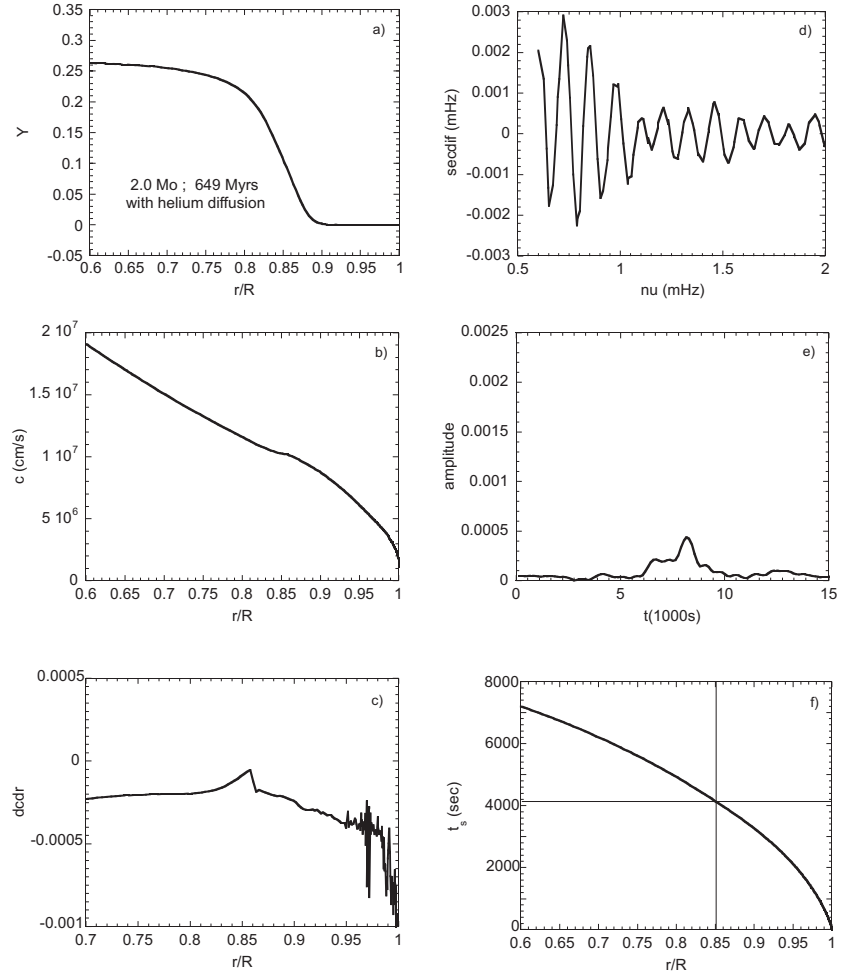

Fig. 5. Same as Fig. 1, for a $2.0 M_{\odot}$ model at $649 \mathrm{Myr}$.

\section{The roAp star HD 60435}

Among all the rapidly oscillating Ap stars which have been detected up to now, only one, HD 60435, presents a frequency spectrum rich enough for the second differences to be computed. This star has been extensively by Matthews et al. (1987) who discovered 17 frequency peaks in the fourier spectrum, which they basically identified as $l=1$ and $l=2$ modes. As for all roAp stars, the amplitudes of the oscillations are modulated according to the rotation period (here 7.7 days). However, contrary to most Ap stars, no magnetic field has yet been clearly detected.

We have computed the observed second differences for this star, using the frequencies given by these authors in their Table 2 (with an uncertainty of $\pm 0.0001 \mathrm{mHz}$ ) and their tentative identification, as given in the same table. For the $0.8428 \mathrm{mHz}$ frequency, we chose the first identification given, i.e $(n-12,2)$ and we assumed that the $1.2848 \mathrm{mHz}$ frequency, which is not clearly identified in their paper, corresponds to $(n-3,1)$.

We present these frequencies and mode identifications in Table 2, with separate columns for $l=1$ and $l=2$. The second differences can only be computed when at least three consecutive modes have been identified for a given $l$. Table 3 gives the computed second differences for HD 60435 and the $l$ value of the corresponding mode. The obtained values for both $l$ are presented in Fig. 7. The modes are labelled with their $l$ value.

The second differences clearly show a modulated trend similar to those presented in Figs. 1 and 4, characteristic of the presence of a helium gradient below the surface. The fourier transform of the observed second differences is presented in Fig. 8 (thick curve) together with those computed for $1.6 M_{\odot}$ 

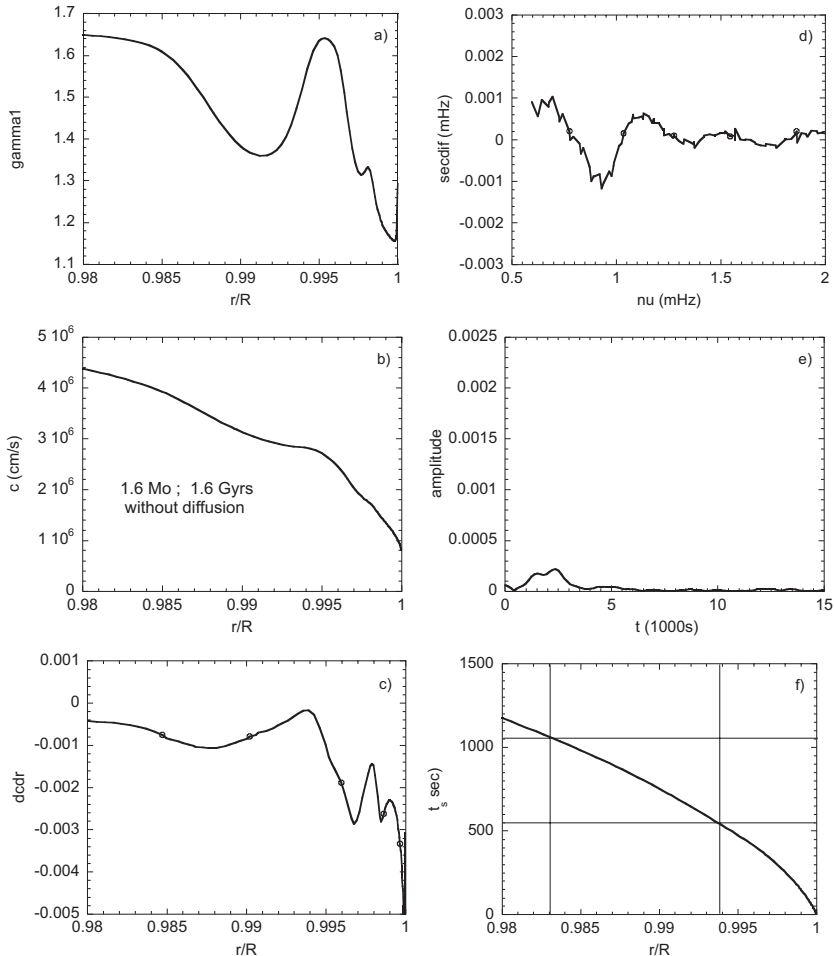

Fig. 6. This figure is similar to Fig. 3 when element diffusion is suppressed, in a $1.6 M_{\odot}$ at $1.6 \mathrm{Gyr}$; here graph a) represents the $\Gamma_{1}$ coefficient which shows a clear feature at the place of the HeII ionisation region; the other graphs are similar to those of Fig. 1; we can clearly see the influence of the helium ionisation regions on the frequencies. Note that the effect is much smaller than that of a helium gradient when diffusion is taken into account.

(95 Myr) and 2.0 $M_{\odot}(63 \mathrm{Myr})$. We can see that the observed peak for HD 60435 lies at a position close to those of the two models. The maximum is found at a period of $3400 \mathrm{~s}$, which corresponds to an acoustic depth of the helium gradient $t_{s} \simeq 1700 \mathrm{~s}$. With a spectral classification A3V, this star should have a mass close to $2.0 M_{\odot}$. We can deduce from this study that the modulation observed in the second differences for the star HD 60435 may be a signature of a helium gradient at a radial depth of about $90 \%$ to $95 \%$ of the stellar radius.

The uncertainties in the frequency measurements lead to relative uncertainties of the second differences smaller than $10 \%$. Simulations of the error in the position of the amplitude peak due to the uncertainties in the frequencies show that the results are quite robust: the time $t_{s}$ at the maximum does not change by more than $0.05 \%$. A more important uncertainty arises from the small number of observed frequencies. A reasonable estimate of this uncertainty is obtained by assuming that a new maximum could appear between the presently observed one and the two adjacent points if more frequencies were observed. Such an estimate leads to $\pm 300 \mathrm{~s}$ in the Fourier transform, or: $t_{s}=1700 \pm 150 \mathrm{~s}$.

These results relie on the mode identification which is given as "tentative" by Matthews et al. (1987). Note however that the difficulty in the identification was due to the fact that the frequencies were not exactly at the expected values. The present
Table 2. Observed frequencies and mode identifications in HD 60435.

\begin{tabular}{lrll}
\hline \hline$v(\mathrm{mHz})$ & mode id.: & $v(\mathrm{mHz})$ & mode id. \\
\hline 0.7090 & $(n-14,1):$ & 0.8428 & $(n-12,2)$ \\
0.7614 & $(n-13,1):$ & 0.9397 & $(n-10,2)$ \\
& $:$ & 0.9906 & $(n-9,2)$ \\
1.1734 & $(n-5,1):$ & 1.0433 & $(n-8,2)$ \\
1.2250 & $(n-4,1):$ & 1.0990 & $(n-7,2)$ \\
1.2848 & $(n-3,1):$ & 1.1482 & $(n-6,2)$ \\
1.3281 & $(n-2,1):$ & 1.3525 & $(n-2,2)$ \\
1.3810 & $(n-1,1):$ & 1.4073 & $(n-1,2)$ \\
1.4334 & $(n, 1):$ & 1.4572 & $(n, 2)$ \\
\hline
\end{tabular}

Note: after Matthews et al. (1987); left columns: $l=1$ right columns: $l=2$.

Table 3. Computed second differences for HD 60435.

\begin{tabular}{lll}
\hline \hline$v(\mathrm{mHz})$ & Sec. Dif. $(\mathrm{mHz})$ & $l$ \\
\hline 0.9906 & +0.0018 & 2 \\
1.0433 & +0.0030 & 2 \\
1.0990 & -0.0065 & 2 \\
1.2250 & +0.0082 & 1 \\
1.2848 & -0.0016 & 1 \\
1.3281 & +0.0096 & 1 \\
1.3810 & -0.0005 & 1 \\
1.4073 & -0.0049 & 2 \\
\hline
\end{tabular}

study shows that the observed frequencies are consistent with the presence of a helium gradient.

\section{Conclusion}

We have computed models of $1.6 M_{\odot}$ and $2.0 M_{\odot}$ main sequence stars with and without helium diffusion and we have shown that asteroseismology can give clear signatures of the presence of helium gradients below the surface in these A-type stars. The analyses of the second differences in the oscillation frequencies show modulations which are due to the partial reflection of the acoustic waves at the place of the helium gradient.

At the present time these theoretical results can be compared to the observations of only one star, namely the roAp HD 60435. The computations of the second differences can only been done if enough modes with adjacent values of $n$ are detected for the same value of $l$. For HD 60435, with the presently detected modes, we could compute $8 \mathrm{~s}$ differences, 


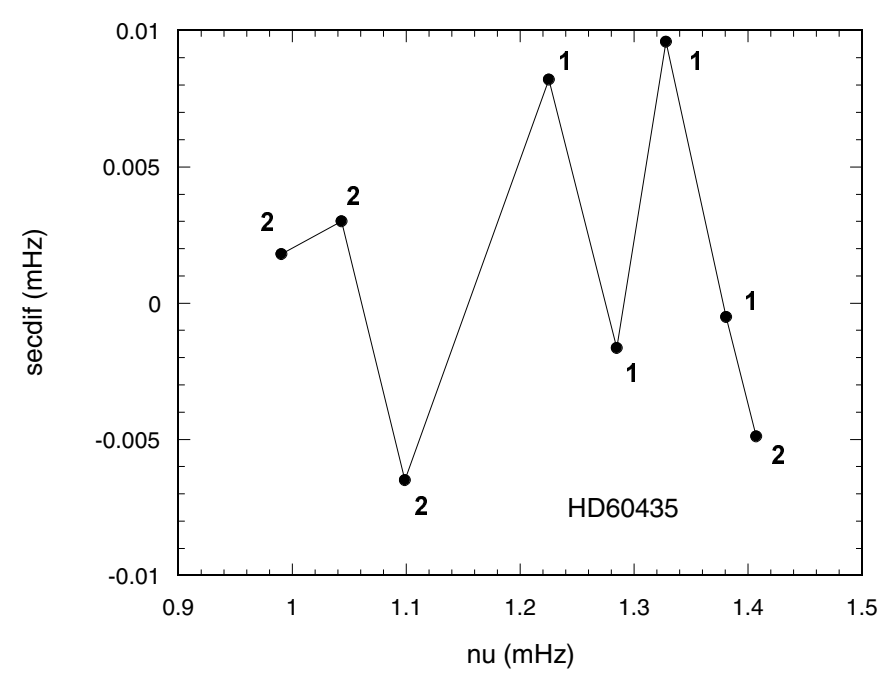

Fig. 7. Second differences in the oscillation frequencies of HD 60435 as found from Matthews et al. (1987); the points are labelled with their $l$ values.

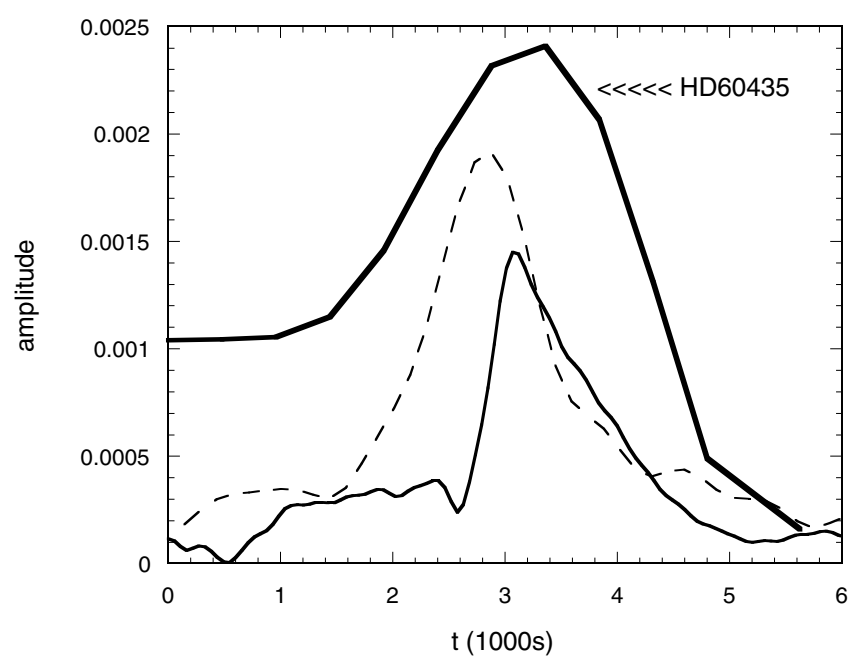

Fig. 8. Fourier transform of the second differences of HD 60435 (Fig. 7) in heavy solid line; the dashed curve represents the computed fourier transforms of the $1.6 M_{\odot}, 95 \mathrm{Myr}$ model and the light solid curve represents that of the $2.0 M_{\odot}, 63 \mathrm{Myr}$ model.

which enabled us to give evidence of a modulation similar to the one expected in case of the presence of a helium gradient below the surface. Furthermore, we could derive the acoustic depth of this gradient: $1700 \pm 150 \mathrm{~s}$. The corresponding fractional radius is model-dependent, but from our computations we suggest that it should lie around $r / R \simeq 0.9$ to 0.95 .

In our evolutionary sequences, which are computed with pure microscopic diffusion, a helium gradient at this depth is obtained early in the stellar life (65 Gyr). However, in real stars microscopic diffusion must compete with macroscopic motions so that the helium gradient can develop later in the evolution: our strong result for HD 60435 consists in determining the acoustic depth of the helium gradient, not the age of the star.
As for all roAp stars, the amplitude of the oscillations observed in this star is modulated according to the rotation period, of 7.7 days. This modulation is probably related to the presence of a magnetic field, although no clear detection have been obtained. Our theoretical computations have been done for spherically symetrical stars, without rotation-induced mixing and ignoring any magnetic effect. In the future, the influence of the magnetic structure of the stars will have to be studied in detail. New models will also be computed, including the abundance variations of heavy elements (with computations of radiative accelerations), which have been neglected here.

The results we have obtained are consistent with the idea that helium diffusion does occur in roAp stars. It has been suggested in the past that the oscillations observed in these stars could be triggered by helium $\kappa$-mechanism; such a process could occur only if helium accumulated due to the combined effect of diffusion and a hypothetical stellar wind (Vauclair et al. 1991). More recently Balmforth et al (2001) showed that such a helium accumulation was unable to destabilise the stars, while the triggering could be due to the hydrogen $\kappa$-mechanism, enhanced in the polar regions in case of pure helium diffusion. The present results are strongly in favor of this model.

In the future, new models will be computed, which will include the abundance variations of heavy elements (with computations of radiative accelerations). The influence of the magnetic structure of the stars will also have to be studied in details.

It will be interesting to try to detect as many modes as possible in roAp stars to make similar comparisons with other stars. Few modes are generally detected in these stars, probably due to mode trapping. New instruments and techniques may help in detecting other modes. The frequencies should be determined with a precision of order $0.1 \mu \mathrm{Hz}$ for the second differences to be computed with a relative uncertainty of order $10 \%$ and the method described here to be applicable. HD 60435 should also be studied in more detail with the most recent techniques to obtain a higher precision of the results.

Acknowledgements. We thank the referee whose interesting critical remarks and comments strongly helped improving this paper. We warmly thank Hiromoto Shibahashi and Don. W. Kurtz for stimulating discussions. We also thank Stephane Charpinet who provided his code for the computation of the oscillation frequencies and Noel Dolez who provided his slow Fourier transform code. Sylvie Vauclair acknowledges a grant from Institut universitaire de France and Sylvie Théado the grant POCTI/1999/FIS/34549 approved by FCT and POCTI, with funds from the European Community programme FEDER.

\section{References}

Balmforth, N. J., Cunha, M. S., Dolez, N., Gough, D. O., \& Vauclair, S. 2001, Mon. Not. R. Astron. Soc., 323, 362

Dziembowski, W. A., \& Goode, P. R. 1996, ApJ, 458, 338 
Gelbmann, M., Ryabchikova, T., Weiss, W. W., et al. 2000, A\&A, 356, 200

Gough, D. O. 1990, in Progress of Seismology of the Sun and Stars, Proc. Oji International Seminar Hakone (Japan: Springer Verlag), Lect. Notes Phys., 367, 283

Kurtz, D. W. 1990, ARA\&A, 28, 607

Matthews, J. M., Kurtz, D. W., \& Wehlau, W. H. 1987, ApJ, 313, 782

Mazumdar, A., \& Antia, H. M. 2001, A\&A, 377, 192

Michaud, G. 1970, ApJ, 160, 641

Miglio, A., Christensen-Dalsgaard, J., Di Mauro, M. P., Monteiro, M. J. P. F. G., \& Thompson, M. J. 2003, in Asteroseismology across the HR diagram, ed. M. J. Thompson, M. S. Cunha, \& M. J. P. F. G. Monteiro (Dordrecht: Kluwer), 537

Monteiro, M. J. P. F. G., Christensen-Dalsgaard, J., \& Thompson, M. J. 1994, A\&A, 283, 247
Monteiro, M. J. P. F. G., \& Thompson, M. J. in New eyes to see inside the Sun and stars (Dordrecht: Kluwer), ed. F.-L. Deubner, J. Christensen- Dalsgaard, \& D. W. Kurtz, Proc. IAU Symp., 185, 317

Richard, O., Vauclair, S., Charbonnel, C., \& Dziembowski, W. A. 1996, A\&A, 312, 1000

Richard, O., Michaud, G., \& Richer, J. 2001, ApJ, 558, 377

Richard, O., Théado, S., \& Vauclair, S. 2004, Sol. Phys., in press

Roxburgh, I. W., \& Vorontsov, S. V. 2001, MNRAS, 322, 85

Théado, S., \& Vauclair, S. 2003, ApJ, 587, 795

Vauclair, S. 1975, A\&A, 45, 233

Vauclair, S. 2003, Ap\&SS, 284, 205

Vauclair, G., \& Vauclair, S. 1982, ARA\&A, 20, 37

Vauclair, S., Dolez, N., \& Gough, D. O. 1991, A\&A, 252, 618 